\title{
La innovación en los centros de enseñanza de Bachillerato: análisis realizado en la Comunidad Valenciana
}

\author{
Rubén J. Cuñat Giménez \\ Universitat de València. España. \\ ruben.cunat@uv.es
}

Recibido: 28/5/2020

Aceptado: 30/9/2020

Publicado: 5/7/2021

\section{Resumen}

La capacidad para innovar en un establecimiento escolar depende de los recursos disponibles y utilizados para contribuir a generar nuevas prácticas en su funcionamiento. En el presente trabajo se analizan dichos recursos en los centros de Bachillerato de la Comunidad Valenciana, así como la influencia que pueden ejercer su tamaño y su antigüedad sobre la innovación. La información se ha obtenido a partir de un cuestionario enviado a las instituciones de educación en una escala tipo Likert. La muestra se compone de 71 centros (55 públicos y 16 privados), y los resultados presentan las valoraciones que hacen respecto a la presencia de los recursos de innovación en su institución, la comparativa entre centros públicos y centros privados y el análisis sobre la posible influencia que ejerce su tamaño, así como su antigüedad, para cada una de las variables estudiadas. Solo la variable relacionada con la disposición de materiales didácticos y multimedia muestra una ligera diferencia entre centros públicos y centros privados en cuanto a su capacidad para innovar, mientras que se constata que el tamaño del establecimiento no condiciona la renovación. En cuanto a su antigüedad, es la variable referida al profesorado la que marca la diferencia entre ambos tipos de centros.

Palabras clave: innovación; Bachillerato; centro de enseñanza; tamaño del centro; antigüedad del centro

Resum. La innovació en els centres d'ensenyament de batxillerat: anàlisi a la Comunitat Valenciana

La capacitat per a innovar en un establiment educatiu depèn dels recursos disponibles i utilitzats per a contribuir a generar noves pràctiques en el seu funcionament. En el present treball s'hi analitzen aqueixos recursos en els centres de batxillerat de la Comunitat Valenciana, així com la influència que poden exercir la grandària i l'antiguitat sobre la innovació. La informació ha estat obtinguda a partir d'un qüestionari enviat a les institucions d'educació en una escala tipus Likert. La mostra es compon de 71 centres ( 55 de públics i 16 de privats), i els resultats presenten les valoracions que fan respecte a la presència dels recursos d'innovació a la seua institució, la comparativa entre centres públics i centres privats i l'anàlisi sobre la possible influència que exerceix la grandària, així com l'antiguitat, en cadascuna de les variables estudiades. Només la variable relacionada amb la disposició de materials didàctics i multimèdia mostra una lleugera diferència entre centres públics i 
centres privats quant a la capacitat que té per a innovar, mentre que es constata que la grandària de l'establiment no en condiciona la renovació. Quant a l'antiguitat, és la variable referida al professorat la que marca la diferència entre tots dos tipus de centres.

Paraules clau: innovació; batxillerat; centre d'ensenyament; grandària del centre; antiguitat del centre

\section{Abstract. Innovation in high schools: Analysis in the Valencian Community}

The ability to innovate in an educational institution depends on the available resources and how they are used to develop new practices in the school's functioning. In this paper, these resources are analyzed in high schools of the Valencian Community, as well as the influence that the size of the school and its age can have on innovation. The information was obtained by means of a Likert-type questionnaire, which was sent to the schools. The sample consists of 71 schools ( 55 public and 16 private). The results present the schools' assessment of innovation resources, the comparison between public and private institutions, and the analysis of the possible influence of the size and age of the schools for each of the variables studied. Only the variable related to the availability of teaching and multimedia materials shows a slight difference between public and private schools in their capacity to innovate, while school size does not influence innovation. As for school age, the variable "teaching staff" marks the difference between the two types of schools.

Keywords: innovation; high school; educational institution; school size; school age
Sumario
1. Introducción
4. Resultados
2. Los recursos de innovación en un
5. Discusión y conclusiones
centro educativo
Referencias bibliográficas

3. Método

\section{Introducción}

La innovación consiste en institucionalizar las mejores prácticas y asumir compromisos que garanticen su permanencia en el tiempo (Rodríguez y Gairín, 2015). Uno de los principales errores cuando nos referimos a la innovación es asociarla de forma exclusiva a los avances tecnológicos o a la invención (Rahmat, 2020), puesto que esta va más allá (Serdyukov, 2017), por ello debemos verla como una nueva forma de hacer las cosas (Jewitt, Xambo y Price, 2017).

El movimiento de innovación y mejora de los centros docentes nació a finales de la década de 1960 (Murillo, 2003), y en las décadas de 1970 y 1980 se vinculó a un movimiento innovador que puso énfasis en el protagonismo de los docentes. A partir de la década de 1990 y principalmente en los primeros diez años del presente siglo, con la descentralización y la autonomía de los centros educativos, se vinculó a la incorporación de nuevas tecnologías de la información y la comunicación (TIC) y al protagonismo de las instituciones educativas como promotoras del cambio "desde abajo» (Unesco, 2016). 
En este sentido, cualquier innovación debería conseguir la mejora de resultados, tanto en profesores como en alumnado, empleando el mismo esfuerzo que antes de la innovación a través del desarrollo de su capacidad para innovar. Hablamos de capacidad de innovación como la habilidad de una organización para provocar y desarrollar un ambiente de cambio, teniendo en cuenta que los ámbitos para la innovación en la enseñanza no son compartimentos estancos, ya que existe una estrecha interdependencia entre ellos: aula, centro escolar, Administración y comunidad (Rosales, 2012). Es importante considerar, por tanto, que dentro del aula se deben impulsar modificaciones pedagógicas a través de las TIC (González y De Pablos, 2015), sabiendo que los problemas de equipamiento son uno de los principales obstáculos para no utilizarlas (Area, 2005). Además, el centro escolar debe promover la innovación para la mejora continua (Pericacho, 2014), y la Administración y la comunidad deben participar de forma activa en dicho proceso (Gil, Antelm y Cacheiro, 2018).

Este trabajo analiza el grado en el que los centros educativos de Bachillerato públicos y privados de la Comunidad Valenciana están orientados hacia actividades de innovación a partir de los recursos disponibles y utilizados en ellos.

Por otra parte, se realiza un análisis empírico que determina si hay diferencias significativas entre los centros públicos y los privados en cuanto a las variables de innovación, utilizando como modelo estadístico una prueba $t$ de Student para la igualdad de medias.

Finalmente, se estudia el efecto que ejerce el tamaño del centro, por una parte, y la antigüedad, por otra, sobre la innovación. El fundamento para la realización de este tipo de contraste se centra en analizar si se pueden considerar estadísticamente significativas las diferencias entre las medias en las variables cuantitativas de innovación para los subgrupos definidos de tamaño y antigüedad, siendo la prueba de significación tradicionalmente aplicada en este caso la conocida como análisis de varianza (ANOVA) (Fisher, 1935).

\section{Los recursos de innovación en un centro educativo}

Las organizaciones educativas presentan innovación cuando ofrecen productos o servicios nuevos o mejorados, tales como libros de texto o recursos educativos, pero especialmente cuando: 1) incorporan de forma significativa nuevos procesos modificados para la prestación de sus servicios (recursos pedagógicos, tecnológicos...); 2) practican nuevas formas de organizar sus actividades cambiando la forma de trabajo, tanto de profesores como de alumnado, y 3) utilizan nuevas técnicas de comunicación o de relaciones externas con el entorno y las administraciones (Vincent-Lancrin et al., 2019).

El análisis de la capacidad que tiene un centro educativo de cambiar implica identificar las dimensiones implícitas en los procesos innovadores. En este sentido, García-Valcárcel y Tejedor (2010) identifican la organización del centro, el profesorado, la enseñanza (metodología didáctica) y el aprendizaje del 
alumnado como dimensiones de análisis, haciéndolas operativas a través de diversas variables o indicadores.

Por su parte, Berends y King (1994) señalan el profesorado, la gestión, el liderazgo del centro y la comunidad de recursos como dimensiones respecto a la innovación de los estudiantes. Parrilla (2000), citado en Rodríguez y Gairín (2015), a partir de otros estudios nacionales e internacionales, señala como factores claves en el proceso de aprendizaje organizativo e innovación a las características externas del centro (entorno y grupos de interés), a las características internas (proyecto educativo, liderazgo, recursos físicos disponibles...) y a las características de las aulas (asunción de un currículo común para todos los estudiantes, metodologías flexibles, preparación del profesorado...), orientadas a potenciar un contexto que facilite un aprendizaje organizativo a partir de los recursos (humanos, materiales y funcionales) (Rodríguez y Gairín, 2015; Marquès, 2005), junto a un clima y a una cultura favorables.

Por tanto, no puede haber innovación en un centro educativo sin la presencia de un contexto que la favorezca y la disposición de recursos para que esta se pueda implementar.

En este sentido, por lo que respecta a los recursos y siguiendo las indicaciones del profesor Pere Marquès (2005), estos deberían incluir:

\section{a) Recursos materiales}

- Las infraestructuras y los modelos tecnológicos de uso de las TIC, por lo que se refiere a la disposición de recursos tecnológicos correctamente ubicados e instalados, incluyendo los programas y los medios digitales necesarios, así como el adecuado mantenimiento en cada centro; siendo los ordenadores, internet y las pizarras digitales interactivas las tecnologías más utilizadas (Colás, De Pablos y Ballesta, 2018). Las TIC aportan la posibilidad de flexibilizar y mejorar procesos que inciden directamente en el aprendizaje (González y De Pablos, 2015).

- Materiales de apoyo para la educación, incluyendo recursos didácticos como libros, juegos, vídeos, materiales multimedia y del ciberespacio, que faciliten el trato personalizado al alumnado, además de plataformas de contenidos en red.

\section{b) Recursos humanos}

Están relacionados con las personas y su implicación, y contribuyen a crear un clima de aceptación de las tecnologías que permite mejorar los procesos de aprendizaje (Fernández, Fernández y Rodríguez, 2018). En este sentido los podemos identificar en los siguientes participantes:

- El profesorado. Su preparación es fundamental para garantizar la adecuada introducción de prácticas innovadoras en el aula (Fernández y Fernández, 2016), sin embargo, su rol tradicional basado en la transmisión de cono- 
cimientos de forma rutinaria no es suficiente para poder cubrir las demandas educativas actuales (Ferrández y Sánchez, 2014).

- El equipo directivo. Incorpora la preparación de este colectivo en cuanto a la función directiva, además de la función de liderazgo en la organización (Cárdenas, Farías y Méndez, 2017). Por otra parte, debe generar una cultura colaborativa que permita orientar las acciones individuales y las colectivas para movilizar el potencial personal y profesional de los componentes de la organización (Gairín y Rodríguez, 2011).

- El personal no docente. Funcionamiento y logística del centro.

- El alumnado. Grado de motivación y disposición a pasar de un papel pasivo e individualista a otro activo y de tipo cooperativo (De la Torre y Herasme, 2014).

- Las familias. El centro escolar y los progenitores permiten realizar el intercambio de informaciones y formas de pensar para el desarrollo del aprendizaje del alumnado (Gil, Antelm y Cacheiro, 2018).

- El entorno social. Lo componen las entidades educativas, culturales y deportivas que pueden contribuir al desarrollo de los estudiantes.

\section{c) Recursos funcionales}

Es importante la existencia de un proyecto educativo en el que se den a conocer los valores que se quieren transmitir y que incorpore un plan de innovación. Un proyecto que sea asumido por todos y que estos se sientan implicados en su realización (Gairín, 2000).

\section{Método}

\subsection{Diseño}

El cuestionario se diseñó en base a las proposiciones teóricas que figuran en este artículo respecto a los recursos que ejercen un impacto sobre la innovación en un centro educativo, haciéndolos operativos a partir de variables o indicadores (tabla 1) y utilizando una escala Likert 1-5 (donde 1 se refiere a desacuerdo total y 5, a acuerdo total). Se confeccionó un cuestionario con 37 ítems.

Para medir el tamaño del centro se consideraron dos criterios:

- Tamaño del centro con relación al número de profesores y de profesoras que imparten clases en Bachillerato: 1) entre 20 y 50, y 2) más de 50.

- Tamaño del centro con relación al número de estudiantes de Bachillerato. Se definieron 5 categorías: 1) centros de menos de 30 alumnos; 2) centros de entre 30 y 60 alumnos; 3) de entre 60 y 100; 4) de entre 100 y 200 , y 5) de más de 200 alumnos.

La categorización de la variable antigüedad fue la siguiente: 1) fundación antes de 1970; 2) entre 1971 y 1995, y 3) después de 1996. 
Tabla 1. Recursos y variables para la innovación

\begin{tabular}{lll}
\hline Tipo de recurso & Variable o indicador & Contenidos \\
\hline $\begin{array}{l}\text { Recursos mate- } \\
\text { riales (15 ítems) }\end{array}$ & $\begin{array}{l}\text { Objetivos y estructura } \\
\text { de la formación }\end{array}$ & $\begin{array}{l}\text { Analizan la disposición de materiales didácticos } \\
\text { actualizados, materiales multimedia y ciberespacio. }\end{array}$ \\
& Metucturas físicas & $\begin{array}{l}\text { Aspectos relacionados con el acceso a Internet, a } \\
\text { los medios tecnológicos disponibles y a los recur- } \\
\text { sos digitales. }\end{array}$ \\
$\begin{array}{l}\text { Recursos huma- } \\
\text { nos (18 ítems) }\end{array}$ & $\begin{array}{l}\text { Formación y apoyo al } \\
\text { profesorado } \\
\text { aspectos de evaluación y seguimiento del alum- } \\
\text { nado. }\end{array}$ & $\begin{array}{l}\text { Por una parte, recoge el nivel de preparación del } \\
\text { profesorado para la aplicación de nuevas metodolo- } \\
\text { gías y, por otra, el apoyo que recibe de la dirección } \\
\text { para la aplicación de estas. }\end{array}$ \\
\hline $\begin{array}{l}\text { Incorporan la participación de las familias en los } \\
\text { aspectos propios de la innovación del centro, así } \\
\text { como su implicación. }\end{array}$ \\
$\begin{array}{l}\text { Recursos } \\
\text { funcionales (4 } \\
\text { ítems) }\end{array}$ & Aspectos organizativos de las & $\begin{array}{l}\text { Relacionado con los planes de innovación en mar- } \\
\text { cha y futuros del centro, así como el sistema de } \\
\text { control de los mismos. }\end{array}$ \\
\hline
\end{tabular}

Fuente: elaboración propia a partir de Marquès (2005).

\subsection{Población y muestra}

La población objeto de estudio queda definida por el conjunto de centros educativos de Bachillerato registrados en la base de datos pública por la Consejería de Educación, Investigación y Deporte de la Generalidad Valenciana en el mes de octubre de 2018 (331 centros públicos y 140 privados). Se envió un cuestionario vía correo electrónico a cada uno de estos centros y se obtuvieron 71 respuestas de noviembre a enero de 2019 (55 de centros públicos y 16 de centros privados), siendo esta la muestra definitiva objeto de examen.

Un análisis descriptivo del perfil de las instituciones educativas de la muestra (tabla 2) indica que el 49,3\% de las que se estudiaron fueron creadas entre los años 1976 y 2000 , y que en su mayor parte se trata de centros públicos $(77,5 \%)$. Casi la mitad se ubica en la provincia de Valencia y el mayor número de ellos corresponde a centros donde asisten entre 60 y 100 alumnos. La población total de centros de Bachillerato en la Comunidad Valenciana se reparte en un $70,28 \%$ que son públicos y un $29,72 \%$, privados; un 53,29\% de los cuales corresponde a la provincia de Valencia; un $34,61 \%$, a la de Alicante, y un 12,10\%, a la de Castellón. Dichos porcentajes no difieren sustancialmente respecto al tamaño de la muestra analizada, por lo que esta se puede considerar proporcional en cuanto al tamaño de la población. 
Tabla 2. Perfil de la muestra de los centros educativos de Bachillerato en la Comunidad Valenciana

\begin{tabular}{|c|c|}
\hline Fecha de constitución del centro & $\begin{array}{l}\text { El } 23,9 \% \text { fue creado antes de } 1975 \text {; el } 49,3 \% \text {, entre } \\
1976-2000 \text {, y el } 26,8 \% \text {, después de } 2000 \text {. }\end{array}$ \\
\hline Tipo de centro educativo & El $22,5 \%$ son centros privados y el $77,5 \%$, públicos. \\
\hline Ubicación (provincia) & $\begin{array}{l}\text { El } 39,4 \% \text { se encuentra en Alicante; el } 12,7 \% \text {, en Cas- } \\
\text { tellón, y el } 47,9 \% \text {, en Valencia. }\end{array}$ \\
\hline $\begin{array}{l}\text { Número total de estudiantes de Bachi- } \\
\text { llerato }\end{array}$ & $\begin{array}{l}\text { En el } 2,8 \% \text { asisten menos de } 30 \text { alumnos; en el } \\
14,1 \% \text {, entre } 30 \text { y } 60 \text {; en el } 29,6 \% \text {, entre } 60 \text { y } 100 \text {; en } \\
\text { el } 38 \% \text {, entre } 100 \text { y } 200 \text {, y en el } 15,5 \% \text {, más de } 200 \text {. }\end{array}$ \\
\hline
\end{tabular}

Fuente: elaboración propia.

\subsection{Instrumentos}

Los datos fueron analizados con el programa SPSS, versión 26. La consistencia del cuestionario fue validada calculando el coeficiente Alfa de Cronbach para cada bloque de la escala Likert. Como se puede ver en la tabla 3, su valor para los ítems analizados es de 0,923 , lo que representa una elevada fiabilidad en su consistencia interna.

Tabla 3. Validación del cuestionario realizado

\begin{tabular}{cccc}
\hline & $\begin{array}{c}\text { Alfa de } \\
\text { Cronbach }\end{array}$ & $\begin{array}{c}\text { Alfa de Cronbach basada en } \\
\text { elementos estandarizados }\end{array}$ & $\begin{array}{c}\text { Número de } \\
\text { elementos }\end{array}$ \\
\hline Innovación educativa & 0,923 & 0,928 & 37 \\
\hline
\end{tabular}

Fuente: elaboración propia.

\section{Resultados}

\subsection{Análisis descriptivo de los datos obtenidos para centros públicos y para centros privados}

En la tabla 4, las puntuaciones medias que reflejan cada una de las variables objeto de estudio indican que los centros consideran que sus puntos fuertes, en cuanto a recursos de innovación, se centran en la disposición de materiales didácticos y multimedia actualizados, la preparación de su profesorado para la aplicación de nuevas tecnologías junto con el apoyo que reciben por parte de la dirección y las metodologías utilizadas; mientras que los aspectos más débiles están relacionados con la participación de las familias en el centro, las infraestructuras y la implantación de planes de innovación a medio y largo plazo. 
Tabla 4. Análisis descriptivo de la muestra por variables de estudio

\begin{tabular}{lcccc}
\hline Variables de análisis & Tipo de centro & $\boldsymbol{N}$ & Media & $\begin{array}{c}\text { Desviación } \\
\text { estándar }\end{array}$ \\
\hline Objetivos y estructura & Público & 55 & 3,98 & 0,69 \\
\hline Formación y apoyo & Privado & 16 & 3,89 & 0,57 \\
\hline Metodología & Público & 55 & 3,50 & 0,69 \\
\hline Participación de las & Privado & 16 & 3,62 & 0,68 \\
familias & Público & 55 & 3,46 & 0,60 \\
\hline TIC e infraestructuras & Privado & 16 & 3,45 & 0,53 \\
& Público & 55 & 3,10 & 0,64 \\
\hline Aspectos organizativos & Privado & 16 & 3,10 & 0,74 \\
& Público & 55 & 2,92 & 0,66 \\
\hline & Privado & 16 & 3,30 & 0,72 \\
\hline
\end{tabular}

Fuente: elaboración propia.

\subsection{Distribución de la muestra analizada}

$\mathrm{Si}$ se analizan las valoraciones extremas de las puntuaciones de las variables (tabla 5), se pueden ver diferencias notorias entre los centros públicos y los privados, en cuanto que se observa como el $53,2 \%$ de los primeros otorgan una puntuación alta a la tenencia de materiales actualizados, en contra de un $33,4 \%$ de los segundos. Sin embargo, hay un porcentaje mayor de centros privados que reconocen disponer de mejores infraestructuras físicas y recursos tecnológicos.

Tabla 5. Valores extremos de la distribución

\begin{tabular}{lcccc}
\hline & \multicolumn{2}{c}{ Valoración $>\mathbf{4}$} & \multicolumn{2}{c}{ Valoración $<\mathbf{2}$} \\
\hline & Público & Privado & Público & Privado \\
\hline Objetivos y estructura & $53,2 \%$ & $33,4 \%$ & $0 \%$ & $0 \%$ \\
\hline Formación del profesorado & $27,8 \%$ & $33,4 \%$ & $0 \%$ & $0 \%$ \\
\hline Metodología & $26 \%$ & $20 \%$ & $0 \%$ & $0 \%$ \\
\hline Participación de las familias & $13 \%$ & $20 \%$ & $1,8 \%$ & $0 \%$ \\
Aspectos organizativos & $9,3 \%$ & $13,4 \%$ & $33,3 \%$ & $40 \%$ \\
TIC e infraestructuras & $3,8 \%$ & $20 \%$ & $3,7 \%$ & $0 \%$ \\
\hline
\end{tabular}

Fuente: elaboración propia. 


\subsection{Análisis estadistico de los datos}

En esta parte del trabajo se va a analizar si las diferencias entre los centros públicos y privados en cada una de las variables de innovación identificadas son significativas estadísticamente, así como el efecto del tamaño del centro en los recursos disponibles y la influencia que puede tener la antigüedad del colegio sobre la innovación. El análisis de los efectos del tamaño y la antigüedad sobre las variables de innovación se efectuará tomando las dos muestras agrupadas (centros públicos y privados), dado que se ha comprobado que el patrón de comportamiento sigue los mismos parámetros si se consideran las muestras por separado. Por otra parte, la prueba de Kolmogorov-Smirnov indica que todas las variables se distribuyen normalmente.

\subsubsection{Respecto al análisis de las diferencias entre centros públicos y privados en cuanto a las variables de innovación}

Para llevar a cabo este análisis se ha realizado una prueba $t$ de Student para la igualdad de medias en muestras independientes. En primer lugar, la prueba de Levene para la igualdad de varianzas indica que se pueden suponer varianzas iguales, dado que la probabilidad asociada a dicho estadístico para estas dos variables es mayor que 0,05 . Partiendo de la premisa anterior, se analiza el estadístico $t$ con su nivel de significación bilateral. En la tabla 6 se puede observar que, para un valor de $p<0,05$, es poco probable que la diferencia entre las medias para cada una de estas variables observadas entre los centros públicos y los privados sea significativa. Tan solo la variable TIC e infraestructuras se acerca a este nivel de significación, por lo que se puede afirmar que, aunque hay diferencias entre los centros públicos y los privados en cuanto a los recursos de innovación, estas no son estadísticamente significativas. Este hallazgo se muestra acorde con diferentes estudios sobre centros educativos innovadores (Hernando, 2015; Ministerio de Educación, 2013), donde la innovación no guarda relación con el carácter público o privado del centro en general, sino con la disposición y el uso de recursos de innovación a nivel particular de cada centro.

Tabla 6. Comparación de medias entre centros públicos y centros privados

\begin{tabular}{lcccccc}
\cline { 1 - 5 } \multicolumn{1}{c}{ Variables de análisis } & \multicolumn{2}{c}{ Prueba Levene } & \multicolumn{2}{c}{ Análisis estadístico $\boldsymbol{t}$} & \\
\cline { 1 - 5 } \multicolumn{1}{c}{ Varianzas iguales } & $\boldsymbol{F}$ & Sig. & $\boldsymbol{t}$ & Sig. (bilateral) & Dif. medias \\
\hline Objetivos y estructura & 0,534 & 0,467 & 0,465 & 0,643 & 0,08902 \\
\hline TIC e infraestructuras & 0,006 & 0,941 & $-1,976$ & 0,052 & 0,38030 \\
Metodología & 2,284 & 0,135 & 0,089 & 0,930 & 0,01488 \\
Profesorado & 0,129 & 0,721 & $-0,638$ & 0,526 & $-0,12500$ \\
\hline Participación de las familias & 1,276 & 0,263 & $-0,002$ & 0,999 & $-0,00028$ \\
\hline Organización & 0,226 & 0,636 & $-0,657$ & 0,513 & $-0,20871$ \\
\hline
\end{tabular}

Fuente: elaboración propia. 


\subsubsection{Efecto del tamaño y la antigüedad del centro en los recursos de innovación}

El fundamento para la realización de este tipo de contraste se centra en analizar si se pueden considerar estadísticamente significativas las diferencias entre las medias de las variables de innovación para los subgrupos definidos de tamaño y antigüedad.

Tal como se ha comentado en este trabajo, el tamaño del centro se va a medir tanto por el número de profesores y profesoras que imparten Bachillerato en él como por su número de estudiantes.

Asumiendo un nivel de riesgo alfa de 0,05 , se van a contrastar las siguientes hipótesis:

- Para el tamaño según el número de docentes: $H_{0}: \mu_{1}=\mu_{2}$

Tal como se puede observar en la tabla 7, el valor de significación, es decir, la probabilidad de obtener un valor igual o superior al obtenido en la muestra (valores de $F$ en la tabla) siendo cierta la hipótesis nula es superior a 0,05 para todas y cada una de las variables estudiadas entre grupos. Por tanto, se acepta la hipótesis nula y se puede concluir, con un nivel de riesgo del $5 \%$, que el número de profesores que forman parte de un centro educativo de Bachillerato no es determinante en cuanto a la disposición ni al uso de recursos de innovación. En este sentido, es frecuente observar más implicación del profesorado en centros pequeños que en grandes; sin embargo, las resistencias que se pueden encontrar en los centros mayores por parte de algunos profesores operan a modo de filtro catalizador para impulsar a otros (Sánchez y Murillo, 2010).

Tabla 7. ANOVA de un factor para el tamaño del centro (número de docentes)

\begin{tabular}{llllll}
\hline & & S. C. & gl & $\boldsymbol{F}$ & Sig. \\
\hline Objetivos y estructura & Entre grupos & 0,683 & 1 & 1,532 & 0,220 \\
TIC e infraestructuras & Entre grupos & 0,002 & 1 & 0,004 & 0,950 \\
\hline Metodología & Entre grupos & 0,174 & 1 & 0,500 & 0,482 \\
\hline Profesorado & Entre grupos & 0,008 & 1 & 0,016 & 0,899 \\
\hline Participación de las familias & Entre grupos & 0,075 & 1 & 0,170 & 0,682 \\
\hline Organización & Entre grupos & 0,046 & 1 & 0,037 & 0,849 \\
\hline
\end{tabular}

Fuente: elaboración propia.

- Para el tamaño según el número de estudiantes: $H_{0}: \mu_{1}=\mu_{2}=\mu_{3}=\mu_{4}=\mu_{5}$

Tal como se puede observar en la tabla 8 , el valor de significación, es decir, la probabilidad de obtener un valor igual o superior al obtenido en la muestra (valores de $F$ en la tabla), siendo cierta la hipótesis nula, es superior a 0,05 para todas y cada una de las variables estudiadas entre grupos. Por tanto, se acepta 
Tabla 8. ANOVA de un factor para el tamaño del centro (número de alumnos y alumnas)

\begin{tabular}{llcccc}
\hline & & S. C. & gl & $\boldsymbol{F}$ & Sig. \\
\hline Objetivos y estructura & Entre grupos & 0,587 & 4 & 0,314 & 0,868 \\
TIC e infraestructuras & Entre grupos & 2,468 & 4 & 1,293 & 0,282 \\
\hline Metodología & Entre grupos & 2,006 & 4 & 1,477 & 0,219 \\
\hline Profesorado & Entre grupos & 0,487 & 4 & 0,243 & 0,913 \\
\hline Participación de las familias & Entre grupos & 1,262 & 4 & 0,710 & 0,588 \\
\hline Organización & Entre grupos & 5,866 & 4 & 1,211 & 0,315 \\
\hline
\end{tabular}

Fuente: elaboración propia.

la hipótesis nula y se puede concluir, con un nivel de riesgo del 5\%, que el tamaño de un centro de Bachillerato, medido en función del número de alumnado en todo el centro, no influye en la tenencia ni en el uso de recursos para la innovación.

- Para la relación entre la antigüedad del centro y los recursos de innovación: $H_{0}: \mu_{1}=\mu_{2}=\mu_{3}$

En la tabla 9 se puede observar que en la única variable donde se rechaza la hipótesis nula es en profesorado, dado que su valor de significación es de 0,010 , es decir, inferior a 0,05 . Por tanto, esto indicaría que la antigüedad del centro de Bachillerato solo repercute en el grado de preparación del profesorado y el apoyo que recibe para implementar procesos de innovación.

Tabla 9. ANOVA de un factor para la antigüedad del centro

\begin{tabular}{llcccc}
\hline & & S. C. & gl & $\boldsymbol{F}$ & Sig. \\
\hline Objetivos y estructura & Entre grupos & 0,580 & 2 & 0,639 & 0,531 \\
TIC e infraestructuras & Entre grupos & 0,323 & 2 & 0,331 & 0,720 \\
\hline Metodología & Entre grupos & 1,124 & 2 & 1,660 & 0,198 \\
\hline Profesorado & Entre grupos & 4,158 & 2 & 4,890 & 0,010 \\
\hline Participación de las familias & Entre grupos & 0,577 & 2 & 0,654 & 0,523 \\
\hline Organización & Entre grupos & 2,074 & 2 & 0,832 & 0,439 \\
\hline
\end{tabular}

Fuente: elaboración propia.

Para una mejor comprensión sobre el análisis, en la tabla 10 se puede ver la distribución de los datos separados en subgrupos en función de la antigüedad del centro para la variable profesorado. 
Tabla 10. Información estadística sobre los subgrupos de colegios según las variables antigüedad y profesorado

\begin{tabular}{lccccccc}
\hline & $\boldsymbol{N}$ & Media & Desv. & Mín. & Máx. & $<\mathbf{3 , 2 5}$ & $>\mathbf{4 , 5}$ \\
\hline Antes de 1970 & 17 & 3,61 & 0,80 & 2,50 & 5,00 & $40 \%$ & $20 \%$ \\
\hline Entre 1971 y 1995 & 35 & 3,70 & 0,53 & 2,75 & 4,50 & $20 \%$ & $10 \%$ \\
Después de 1996 & 19 & 3,13 & 0,69 & 2,00 & 4,75 & $60 \%$ & $5 \%$ \\
\hline Total & 71 & 3,52 & 0,68 & 2,00 & 5,00 & $40 \%$ & $10 \%$ \\
\hline
\end{tabular}

Fuente: elaboración propia.

Dado que estadísticamente no resulta obvio interpretar a partir del valor de $F$ y su significación cuál es el tamaño del efecto, hemos calculado el valor de eta-cuadrado $\left(\eta^{2}\right)$ corregida, que tiene un valor de 0,094. Este índice, que oscila entre 0 (independencia entre las variables antigüedad y profesorado en nuestro caso) y 1 (relación perfecta entre las variables), indica el porcentaje de variabilidad de la variable respuesta (profesorado) explicado por la variable categórica (antigüedad). Concluyendo diremos que tan solo el 9,4\% de la variabilidad observada en las valoraciones de la innovación con referencia al profesorado se explica por la pertenencia a los diferentes grupos de antigüedad de los colegios analizados.

Para completar esta parte hemos realizado un análisis de comparaciones múltiples a posteriori (o post hoc) para observar qué grupos difieren en sus medias. Dado que se van a comparar todos los posibles pares de medias, la prueba que vamos a aplicar será la HSD de Tukey.

De esta forma, en la tabla 11 se observa que las diferencias de medias son significativas $(p<0,05)$ entre los colegios que fueron creados entre los años 1971 y 1995 frente a los creados después del año 1996, con un nivel de formación y preparación del profesorado mayor para los colegios más antiguos de esta comparativa. Es importante considerar este aspecto, puesto que la carencia de entusiasmo junto a la preparación del profesorado para provocar cambios es un importante obstáculo para la innovación (Hernández y Medina, 2014).

Tabla 11. Prueba HSD de Tukey para la variable profesorado respecto a la antigüedad del centro

\begin{tabular}{|c|c|c|c|c|c|c|}
\hline & & \multirow[b]{2}{*}{$\begin{array}{c}\text { Dif. } \\
\text { medias }\end{array}$} & \multirow[b]{2}{*}{$\begin{array}{l}\text { Desv. } \\
\text { error }\end{array}$} & \multirow[b]{2}{*}{ Sig. } & \multicolumn{2}{|c|}{ Intervalo de confianza 90\% } \\
\hline & & & & & $\begin{array}{c}\text { Lím. } \\
\text { inferior }\end{array}$ & $\begin{array}{c}\text { Lím. } \\
\text { superior }\end{array}$ \\
\hline \multirow[t]{2}{*}{$\begin{array}{l}\text { Antes de } \\
1970\end{array}$} & $\begin{array}{l}\text { Entre } 1971 \\
\text { y } 1995\end{array}$ & $-0,08$ & 0,19 & 0,904 & $-0,48$ & 0,32 \\
\hline & $\begin{array}{l}\text { Después de } \\
1996\end{array}$ & 0,48 & 0,22 & 0,073 & 0,03 & 0,94 \\
\hline \multirow{2}{*}{$\begin{array}{l}\text { Entre } \\
1971 \text { y } \\
1995\end{array}$} & $\begin{array}{l}\text { Antes } \\
\text { de } 1970\end{array}$ & 0,08 & 0,19 & 0,904 & $-0,32$ & 0,48 \\
\hline & $\begin{array}{l}\text { Después } \\
\text { de } 1996\end{array}$ & 0,56 & 0,18 & 0,009 & 0,18 & 0,96 \\
\hline
\end{tabular}

Fuente: elaboración propia. 


\section{Discusión y conclusiones}

La innovación se ha convertido actualmente en un instrumento que permite a las organizaciones avanzar para adecuarse a las nuevas realidades económicas y sociales, y se vincula tanto al concepto de cambio (Pascual, 2019) como a procesos y resultados (Murillo, 2002). Es importante que la innovación siempre persiga la mejora de los resultados, tanto en profesorado como en alumnado, a través de la disposición de recursos y la adecuada formación de sus docentes (Pegalajar y Colmenero, 2017). Debemos afirmar que su análisis es complejo, dado que supone la implantación de nuevos recursos, materiales, acciones y la participación colaborativa de todos sus miembros (Fullan, 2016).

Del trabajo realizado se desprende que, en general, los centros de Bachillerato (tanto públicos como privados) están mejor preparados en lo que se refiere a la disposición de materiales didácticos y multimedia actualizados, la preparación del profesorado para la aplicación de nuevas tecnologías y el apoyo de la dirección para implementar procesos de cambio; sin embargo, constatan mayores debilidades acerca de los recursos relacionados con infraestructuras e implantación de planes de innovación a medio y largo plazo. El déficit en infraestructuras es acorde con los datos del INE, que indican que en el año 1999 el gasto educativo en centros de Educación Secundaria y Formación Profesional (incluye Bachillerato) se situaba en un 1,27\% del PIB, mientras que en el año 2018 esa cifra descendía hasta el 1,17\%, considerando que en estos momentos nos situamos ante mayores necesidades en materia tecnológica. Es por tanto necesario que la tendencia en cuanto a inversión en centros educativos se invierta, para que estos dispongan de recursos adecuados que les permitan innovar, con el objetivo de adaptarse al nuevo entorno.

En este sentido, hay que destacar que uno de los principales obstáculos a la innovación en un centro educativo es la carencia de recursos suficientes, su utilización y su contexto. Por esta razón no existirá innovación en un centro si carece de recursos materiales, humanos y funcionales apropiados, junto con competencias adecuadas de sus docentes (Perrenoud, 2004).

Si se comparan los recursos disponibles entre los centros públicos y los privados, se puede constatar que los primeros disponen de materiales didácticos y multimedia más actualizados que los segundos, sin embargo, indican una disposición de recursos TIC inferior a estos. De hecho, en 2018 los centros públicos disponían de 2,8 ordenadores por estudiante, frente a los 3,9 de los privados (Colás, De Pablos y Ballesta, 2018).

$\mathrm{Al}$ margen de estas diferencias, el resto de las variables observadas muestran comportamientos similares, lo que nos indica que en general el tipo de centro de Bachillerato (público o privado) no es determinante en la disposición ni en el uso de recursos para la innovación en general.

Por otra parte, se ha analizado la conexión entre el tamaño del centro y los recursos para la innovación, y no se ha encontrado evidencia de que ambos aspectos estén relacionados. A nivel general, este hallazgo es acorde con los diferentes estudios que se han realizado en múltiples campos de actividad, 
donde no se percibe consenso sobre la magnitud ni la relación entre el tamaño de la organización y la innovación (Muñoz y Cordón, 2002).

Por último, por lo que respecta a la antigüedad del centro y su repercusión sobre la disposición y la utilización de los recursos de innovación, la formación y preparación del profesorado, junto al apoyo de la dirección para el impulso de procesos de innovación, mostraron pequeñas diferencias entre los centros creados durante los años 1971 y 1995 respecto a los creados después del año 1996. De forma que los centros creados a partir del año 1996 reconocen una menor preparación del profesorado en metodologías innovadoras y apoyo de la dirección para su implementación. No obstante, es importante señalar que en nuestro análisis tan solo la variable antigüedad podía explicar un 9,4\% de las diferencias en cuanto a los docentes, por lo que sería necesario incluir otros elementos de estudio tales como la edad del profesorado, el sexo o su formación académica.

La preparación del profesorado es otro de los aspectos que sustentan la innovación en un centro de enseñanza, sin embargo, aunque nuestras universidades han ido incorporando progresivamente nuevas técnicas de aprendizaje e incrementando prácticas entre los estudiantes, según el informe TALIS (INEE, 2018), el 52\% de los nuevos docentes de Educación Secundaria asegura que no está suficientemente preparado en lo referente al contenido, a la pedagogía ni a la práctica innovadora en la enseñanza en el aula. Es necesario, por tanto, identificar las limitaciones y las dificultades que pueden encontrar los nuevos docentes a la hora de poner en práctica en sus aulas acciones innovadoras y proponer planes formativos del profesorado para la aplicación de la innovación (Monge y Gómez, 2019).

Como toda investigación, este trabajo tiene una serie de limitaciones. Las preguntas del cuestionario fueron enviadas a la dirección del centro, por lo que presumiblemente fue su director o directora quien atendió su respuesta. En este sentido, la información se podría haber enriquecido si otros integrantes del centro hubieran participado en la encuesta. No obstante, hay que tener en cuenta que este estudio tiene carácter cuantitativo y está relacionado con los centros de Bachillerato. Por tanto, se podrían dar resultados diferentes para otro tipo de institución de enseñanza.

La relevancia del tema, junto a la importancia de los efectos de la innovación sobre la mejora en la educación, nos indica que este trabajo puede tener una continuidad, indagando con un análisis cualitativo sobre los motivos que inducen a disponer de más o mejores recursos de innovación en un centro. Por otra parte, sería interesante realizar este estudio sobre instituciones educativas de otras etapas (infantil, primaria, secundaria...), así como en otras comunidades autónomas. 


\section{Referencias bibliográficas}

Area, M. (2005). Tecnologías de la información y comunicación en el sistema escolar: Una revisión de las líneas de investigación. RELIEVE, 11(1), 3-25. <https://doi.org/10.7203/relieve.11.1.4194>

Berends, M. y KING, M.B. (1994). A description of restructuring in nationally nominated schools: Legacy of the iron cage. Educational Policy, 8(1), 28-50. <https://doi.org/10.1177/0895904894008001002>

CÁrdenas, C.; Farías, G.M. y Méndez, G. (2017). ¿Existe relación entre la gestión administrativa y la innovación educativa?: Un estudio de caso en educación superior. Revista Iberoamericana sobre Calidad, Eficacia y Cambio en Educación, 15(1), 19-35. <https://doi.org/10.15366/reice2017.15.1.002>

Colás, M.P.; Pablos, J. de y Ballesta, J. (2018). Incidencia de las TIC en la enseñanza en el sistema educativo español: Una revisión de la investigación. Revista de Educación a Distancia (RED), 18(56). Recuperado de <https://revistas.um.es/red/ article/view/321471>.

Fernández, F.J. y Fernández, M.J. (2016). Los docentes de la Generación Z y sus competencias digitales. Comunicar, 46(XXIV), 97-105. <http://dx.doi.org/10.3916/C46-2016-10>

Fernández, F.J.; Fernández, M.J. y Rodríguez, J.M. (2018). El proceso de integración y uso pedagógico de las TIC en los centros educativos madrileños. Educación XX1, $21(2), 395-416$. $<$ https://doi.org/10.5944/educxx1.17907>

Ferrández, R. y Sánchez, L. (2014). Competencias docentes en secundaria: Análisis de perfiles de profesorado. RELIEVE, 20(1), art. 1. <https://doi.org/10.7203/relieve.20.1.3786>

Fisher, R.A. (1935). The Design of Experiments. Edimburgo: Oliver and Boyd.

Fullan, M. (2016). The New Meaning of Educational Change. 5. ${ }^{\mathrm{a}}$ ed. Nueva York y Londres: Teachers College Press.

GaIrín, J. (2000). Cambio de cultura y organizaciones que aprenden. Educar, 27, 31-85. <https://doi.org/10.5565/rev/educar.245>

GaIrín, J. y Rodríguez, D. (2011). Cambio y mejora en las organizaciones educativas. Educar, 47(1), 31-50. $<$ https://doi.org/10.5565/rev/educar.70>

García-Valcárcel y Tejedor (2010). Evaluación de procesos de innovación escolar basados en el uso de las TIC desarrollados en la Comunidad de Castilla y León. Revista de Educación, 352, 125-147. Recuperado de <http://www.revistaeducacion. educacion.es/re352/re352_06.pdf>.

Gil, A.; Antelm, A. y Cacheiro, M. (2018). Análisis de la capacidad de innovación escolar desde la perspectiva del profesorado de educación secundaria: La escuela como organización que aprende. Educar, 54(2), 449-468. <https://doi.org/10.5565/rev/educar.864>

González, A. y Pablos, J. de (2015). Factores que dificultan la integración de las TIC en las aulas. Revista de Investigación Educativa, 33(2), 401-417. <https://doi.org/10.6018/rie.33.2.198161>

Hernando, A. (2015). Viaje a la escuela del siglo XXI: Asi trabajan los colegios más innovadores del mundo. Recuperado de <https://www.fundaciontelefonica.com/ cultura-digital/publicaciones/476/>. 
Hernández, E. y Medina, R. (2014). Análisis de los obstáculos y barreras para el cambio y la innovación en colaboración en los centros de secundaria: Un estudio de caso. Revista de Investigación Educativa, 32(2), 499-512. <https://doi.org/10.6018/rie.32.2.172041>

INEE (2018). Informe TALIS 2018. Recuperado de <http://www.educacionyfp.gob. es/inee/evaluaciones-internacionales/talis/talis-2018.html $>$.

Jewitt, C.; Хамbo, A. y Price, S. (2017). Exploring Methodological Innovation in the Social Sciences: The body in Digital Environments and the Arts. International Journal of Social Science Research Methodology, 20(1), 105-120. <https://doi.org/10.1080/13645579.2015.1129143>

Marquès, P. (2005). Cambios en los centros educativos: Construyendo la escuela del futuro. Didáctica, Innovación y Multimedia, 1-39. Recuperado de <https://www. raco.cat/index.php/DIM/article/view/56081/65503>.

Ministerio de EduCación (2013). Estudios sobre la innovación educativa en España. Madrid: Ministerio de Educación. Recuperado de <https://sede.educacion.gob. es/publiventa/PdfServlet? pdf=VP14970.pdf\&area=E $>$.

Monge, C. y Gómez, P. (2019). Innovando la docencia desde la formación del profesorado: Propuestas y realidades. Madrid: Síntesis.

Muñoz, M. y Cordón, E. (2002). Tamaño, estructura e innovación organizacional. Revista Europea de Dirección y Economía de la Empresa, 11(3), 103-120.

Murillo, F.J. (2002). La «Mejora de la Escuela»: Concepto y caracterización. En J. Murillo y M. Muñoz-Repiso (eds.). La mejora de la escuela: Un cambio de mira$d a$ (pp. 15-50). Barcelona: Octaedro.

- (2003). El movimiento teórico-práctico de mejora de la escuela. REICE, 1(2). Recuperado de <https://www.redalyc.org/pdf/551/55110206.pdf>.

Pascual, J. (2019). Innovación educativa: Un proceso construido sobre relaciones de poder. Revista Educación, Politica y Sociedad, 4(2), 9-30. Recuperado de <http:// hdl.handle.net/10486/687976>.

Pegalajar, M. del C. y Colmenero, M. de J. (2017). Actitudes y formación docente hacia la inclusión en Educación Secundaria Obligatoria. Revista Electrónica de Investigación Educativa, 19(1), 84-97. <https://doi.org/10.24320/redie.2017.19.1.765>

Pericacho, F.J. (2014). Pasado y presente de la renovación pedagógica en España (de finales del siglo XIX a nuestros días): Un recorrido a través de escuelas emblemáticas. Revista Complutense de Educación, 25(1), 47-67. <http://dx.doi.org/10.5209/rev_RCED.2014.v25.n1.43309>

Perrenoud, P. (2004). Diez nuevas competencias para enseñar. El Marqués, Querétaro: Quebecor World, Gráficas Monte Albán. Recuperado de <https://www.uv.mx/dgdaie/ files/2013/09/Philippe-Perrenoud-Diez-nuevas-competencias-para-ensenar.pdf $>$.

Raнmat, N. (2020). Innovation in Education: Barriers and facilitating factors. European Journal of Education Studies, 0. <http://dx.doi.org/10.46827/ejes.v0i0.2784>

RoDrígUEZ, D. y GAIRÍN, J. (2015). Innovación, aprendizaje organizativo y gestión del conocimiento en las instituciones educativas. Educación, 24(46), 73-90. Recuperado de <http://revistas.pucp.edu.pe/index.php/educacion/article/view/ $12245>$.

Rosales, C. (2012). Contextos de la innovación educativa. Innovación Educativa, 22, 9-21. Recuperado de <https://www.usc.gal/revistas/index.php/ie/article/ view/724/706>. 
SÁnchez, M. y Murillo, P. (2010). Innovación educativa en España desde la perspectiva de grupos de discusión. Profesorado, 14(1), 171-189. Recuperado de $<$ http://www.ugr.es/local/recfpro/rev141ART9.pdf>.

Serdyukov, P. (2017). Innovation in education: What works, what doesn't, and what to do about it? Journal of Research in Innovative Teaching \& Learning, 10(1), 4-33. <https://doi.org/10.1108/JRIT-10-2016-0007>

Torre, E.H. de la y Herasme, R.M. (2014). Análisis de los obstáculos y barreras para el cambio y la innovación en colaboración en los centros de secundaria: Un estudio de caso. Revista de Investigación Educativa, 32(2), 499-512. $<$ https://doi.org/10.6018/rie.32.2.172041>

Unesco (2016). Texto 1: Innovación Educativa. Lima: Unesco. Herramientas de Apoyo para el Trabajo Docente. Recuperado de <https://unesdoc.unesco.org/ark:/48223/ pf0000247005? posInSet $=2 \&$ queryId $=057 \mathrm{fd} 916-334 \mathrm{e}-4 \mathrm{c} 60-87 \mathrm{a} 7-21 \mathrm{~b} 79 \mathrm{c}$ $1 \mathrm{~d} 402 \mathrm{f}>$.

Vincent-Lancrin, S.; URGel, J.; Kar, S. y Jacotin, G. (2019). Measuring Innovation in Education 2019: What Has Changed in the Classroom? París: OECD Publishing. $<$ https://doi.org/10.1787/9789264311671-en> 